\title{
Natural convection of water in a partially porous enclosure with a heat-generating element
}

\author{
Marina Astanina ${ }^{1, *}$, and Mikhail Sheremet ${ }^{1}$ \\ ${ }^{1}$ Tomsk State University, 634050 Tomsk, Russia
}

\begin{abstract}
Paper presents the numeral results of transient natural convection in a partially porous enclosure with a heat-conducting and heatgenerating element. The horizontal walls of the cavity are adiabatic, while the vertical ones are isothermal and have low temperature. A mathematical model formulated in dimensionless variables such as vorticity, stream function and temperature have been solved by means of the finite difference method. The governing parameters are the porous layer thickness and dimensionless time. The effects of these parameters are shown using streamlines, isotherms and average temperature of the heatconducting element. The results demonstrate an opportunity to intensify the passive cooling system using the porous layer.
\end{abstract}

\section{Introduction}

Study of convective heat transfer in different cavities plays an important role for science and engineering. Research of heat transfer in technical systems helps to enhance the energy parameters.

At present, interest in the problems of natural convection is increasing in connection with the appearance of real task in production. Thus, Chen et al. [1] have conducted a numeral investigation for enhanced heat transfer from multiple discrete heated sources in a horizontal channel by metal-foam porous layer. They have showed that an increase in the solid-fluid interfacial heat exchange results in a decrease in the temperatures difference between the solid and fluid phases for fixed Reynolds number, where the porous media tend to reach local thermal equilibrium (LTE) with the fluid and a larger cooling augmentation of heaters is obtained. Astanina et al. [2] have numerically studied natural convection with temperature-dependent viscosity in a porous cavity. They have considered in detail the effect of viscosity variation parameter on heat transfer.

The objective of this study is a numeral analysis of the fluid flow and heat transfer patterns in a square partially porous enclosure with a heated element under the effect of internal heat generation.

\footnotetext{
* Corresponding author: astanina.marina@bk.ru
} 


\section{Mathematical model and numeral method}

Physical model is schematically shown in Figure 1. Consider the natural convection in a square partially porous enclosure with a heat-generating and heat-conducting element. The left and right walls of the cavity are maintained at a constant cooling temperature $T_{c}$, the top and bottom walls are adiabatic. A heat-generating and heat-conducting element is placed in the centre of the bottom wall and has a constant density of volumetric heat flux $q$. The interior of the cavity is divided into two parts, namely, a water saturated porous layer of height $\gamma$ and a clear water under the porous layer. It is assumed, that the thermophysical properties of the medium do not depend on temperature. The fluid is Newtonian, heatconducting and the flow is laminar; the Boussinesq approximation is valid.

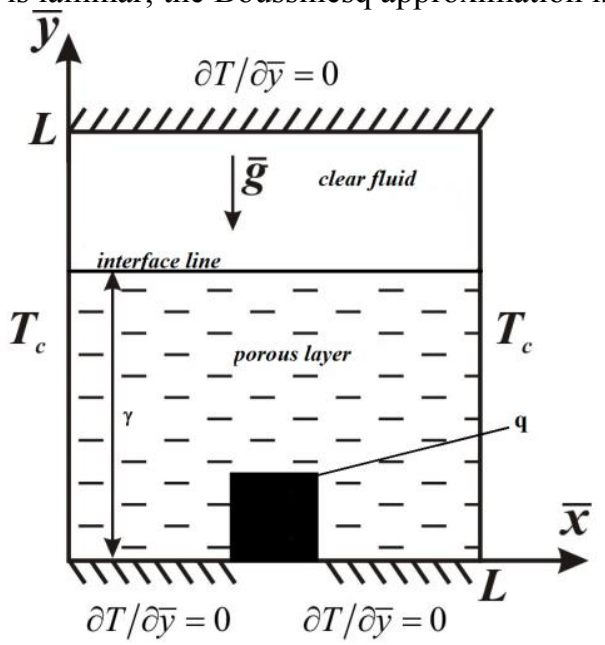

Fig. 1. Physical model.

Under such assumpions the convervation equations for mass, momentum and energy can be written for the two-demensional natural convection in terms « stream function vorticity - temperature » in the following form [2-4]:

- for the clear fluid layer

$$
\begin{gathered}
\frac{\partial^{2} \Psi}{\partial X^{2}}+\frac{\partial^{2} \Psi}{\partial Y^{2}}=-\Omega \\
\frac{\partial \Omega}{\partial \tau}+U \frac{\partial \Omega}{\partial \tilde{O}}+V \frac{\partial \Omega}{\partial Y}=\sqrt{\frac{P r}{R a}}\left(\frac{\partial^{2} \Omega}{\partial \tilde{O}^{2}}+\frac{\partial^{2} \Omega}{\partial Y^{2}}\right)+\frac{\partial \Theta}{\partial X} \\
\frac{\partial \Theta}{\partial \tau}+U \frac{\partial \Theta}{\partial X}+V \frac{\partial \Theta}{\partial Y}=\frac{1}{\sqrt{R a \cdot P r}}\left(\frac{\partial^{2} \Theta}{\partial X^{2}}+\frac{\partial^{2} \Theta}{\partial Y^{2}}\right)
\end{gathered}
$$

- for the porous layer on the basis of the Brinkman-extended Darcy model [2]

$$
\begin{gathered}
\frac{\partial^{2} \Psi}{\partial X^{2}}+\frac{\partial^{2} \Psi}{\partial Y^{2}}=-\Omega \\
\frac{\partial \Omega}{\partial \tau}+U \frac{\partial \Omega}{\partial X}+V \frac{\partial \Omega}{\partial Y}=\sqrt{\frac{P r}{R a}}\left(\varepsilon \frac{\partial^{2} \Omega}{\partial X^{2}}+\varepsilon \frac{\partial^{2} \Omega}{\partial Y^{2}}-\varepsilon^{2} \frac{\Omega}{D a}\right)+\varepsilon^{2} \frac{\partial \Theta}{\partial X}
\end{gathered}
$$




$$
\frac{\partial \Theta}{\partial \tau}+U \frac{\partial \Theta}{\partial X}+V \frac{\partial \Theta}{\partial Y}=\frac{a_{p m} / a_{f}}{\sqrt{R a \cdot P r}}\left(\frac{\partial^{2} \Theta}{\partial X^{2}}+\frac{\partial^{2} \Theta}{\partial Y^{2}}\right)
$$

- for the heat-generating element

$$
\frac{\partial \Theta}{\partial \tau}+U \frac{\partial \Theta}{\partial X}+V \frac{\partial \Theta}{\partial Y}=\frac{a_{h s} / a_{f}}{\sqrt{R a \cdot P r}}\left(\frac{\partial^{2} \Theta}{\partial X^{2}}+\frac{\partial^{2} \Theta}{\partial Y^{2}}+O s\right)
$$

In these equations $P r$ is the Prandtl number, $R a$ is the Rayleigh number, $D a$ is the Darcy number, $O s$ is the Ostrogradsky number. They are defined as follows:

$$
\operatorname{Pr}=\frac{v}{\alpha}, \quad R a=\frac{g \beta \Delta T L^{3}}{\alpha \nu}, \quad D a=\frac{K}{L^{2}}, \quad O s=\frac{q L^{2}}{\lambda_{h s} \Delta T}
$$

The initial and boundary conditions for the equations (1) - (7) are as follows :

$$
\begin{aligned}
& \tau=0: 0 \leq X \leq 1,0 \leq Y \leq 1: \Psi=\Omega=\Theta=0 \\
& \tau>0: X=0,0 \leq Y \leq 1: \Psi=0, \Omega=-\frac{\partial^{2} \Psi}{\partial X^{2}}, \Theta=0 \\
& \tau>0: X=1,0 \leq Y \leq 1: \Psi=0, \Omega=-\frac{\partial^{2} \Psi}{\partial X^{2}}, \Theta=0 \\
& \tau>0: Y=0,0 \leq X \leq 1: \Psi=0, \Omega=-\frac{\partial^{2} \Psi}{\partial Y^{2}}, \frac{\partial \Theta}{\partial Y}=0 \\
& \tau>0: \text { at heat source surface: } \Psi=0, \Omega=-\frac{\partial^{2} \Psi}{\partial n^{2}},\left\{\begin{array}{l}
\Theta_{h s}=\Theta_{f s} \\
\lambda_{p m}
\end{array} \frac{\partial \Theta_{h s}}{\partial \mathrm{n}}=\frac{\partial \Theta_{p m}}{\partial \mathrm{n}}\right. \\
& \tau>0: \text { at internal fluid-porous interface: }\left\{\begin{array}{l}
\Psi_{p m}=\Psi_{f} \\
\frac{\partial \Psi_{p m}}{\partial y}=\frac{\partial \Psi_{f}}{\partial y}\left\{\frac{\partial \Omega_{p m}}{\partial y}=\frac{\partial \Omega_{f}}{\partial y}\left\{\frac{\lambda_{p m}}{\lambda_{f}} \frac{\partial \Theta_{p m}}{\partial y}=\frac{\partial \Theta_{f}}{\partial y}\right.\right.
\end{array}\right.
\end{aligned}
$$

The partial differential equations (1) - (7) with corresponding initial and boundary conditions (9) have been solved by the finite difference method using the uniform grid $[2,4]$. For the approximation of the convective and diffusive terms we used the difference scheme of the second order accuracy. The parabolic equations have been solved on the basis of Samarskii locally one-dimensional scheme [2, 4, 5]. The discretized equations have been solved by Thomas algorithm. The equations for the stream function and have been discretized using the five-point difference scheme on the basis of central differences for the second derivatives. The obtained difference equations have been solved by the successive over relaxation method. Optimum value of the relaxation parameter has been chosen on the basis of computing experiments. 


\section{Results and discussion}

The numeral analysis has been conducted for the following values of the governing parameters: $R a=10^{5}, \operatorname{Pr}=7.0, D a=10^{-3}, O s=10.0, \gamma=0.25-1.0, \mathrm{~T}=0.0-100.0$. The simulation results are illustrated in Figs. 2 and 3.

a)
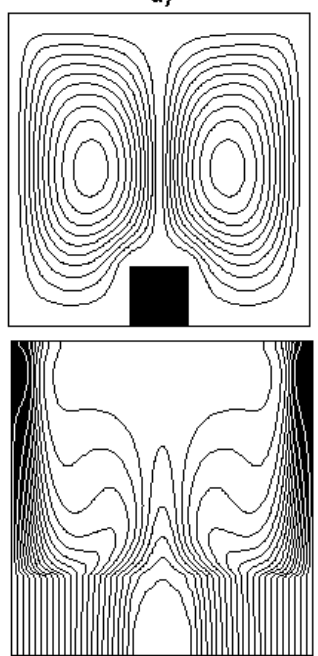

b)
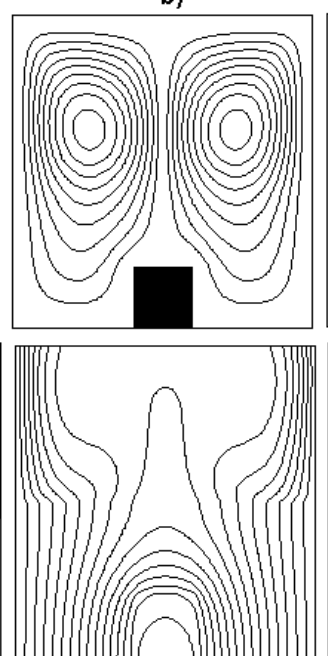

c)
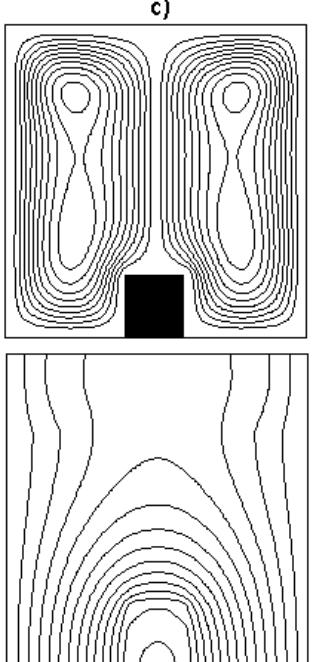

d)
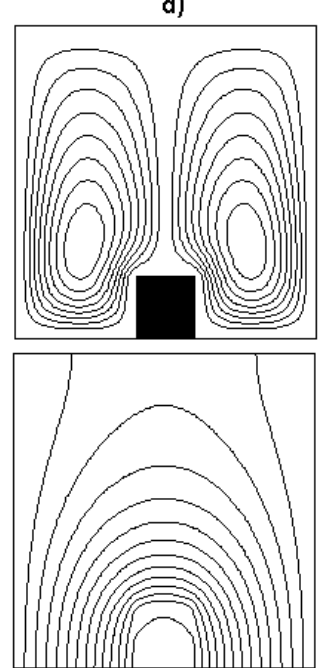

Fig.2. Streamlines and isotherms for $O s=10, D a=10^{-3}: \gamma=0.25-\mathrm{a}, \gamma=0.5-\mathrm{b}, \gamma=0.75-\mathrm{c}$, $\gamma=1.0-\mathrm{d}$

Fig. 2 shows the streamlines and isotherms for different values of the porous layer height $\gamma$. It can be seen, that two convection cells are formed in the cavity, while their cores are located above the interface line, which is clearly seen in Figs. 2a, 2b, 2c. The observed circulations reflect the formation of the ascending flows in the central part of the cavity and the descending ones along the cooling vertical walls. It should be noted, that the heatgenerating element reflects a uniform heating of the cavity in the zone of the porous medium due to low circulation with additional resistance from the solid porous matrix and non-uniform heating in the zone of clear water. In the case of a completely porous enclosure (see Fig. 2d) the cavity is heated uniformly everywhere.

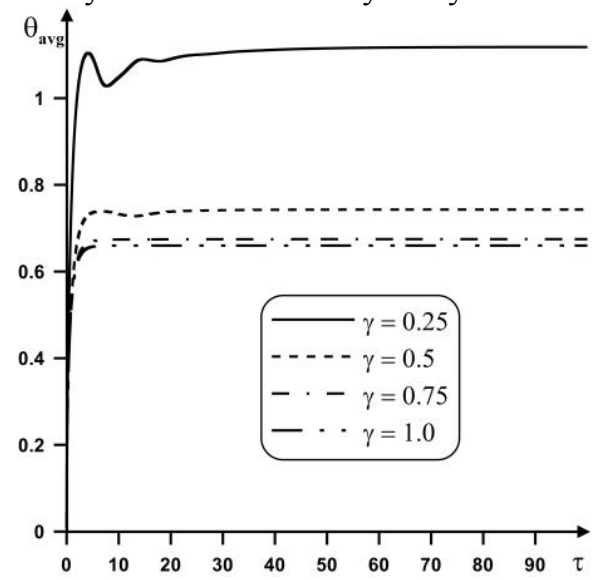

Fig.3. Dependences of the average temperature inside the heater on the porous layer thickness. 
The effect of the porous layer thickness on the average temperature within the heat source is presented in Figure 3. It is possible to conclude that the porous layer allows to intensify the passive cooling system inside the closed domain with a heat-generating element. Moreover, one can find an optimum value of the porous layer thickness to have an effective cooling and optimal fluid flow patterns inside the cavity.

\section{Conclusion}

Numerical simulation of natural convection of water in a square cavity partially filled with a porous medium under the effect of heat-generating and heat-conducting element has been performed. Obtained results have shown that porous insertion can be an effective parameter for an intensification of heat removal from the heat-generating element. Such technique can be used for cooling of electronic elements in the case of impermeable electronic cabinets with one or several heaters.

This work was supported by the Russian Scientific Fond (Project No. 17-79-2014).

\section{References}

1. C.C. Chen, P.C. Huang, H.Y. Hwang, Int. J. Heat Mass Transf., 58, (2013), DOI: 10.1016 / j.ijheatmasstransfer.2012.11.041

2. M.S. Astanina, M.A. Sheremet, J.C. Umavathi, Transp. Porous Media, 110, 1 (2015), DOI: $10.1007 / \mathrm{s} 11242-015-0558-\mathrm{x}$

3. D.A. Nield, A. Bejan, Convection in Porous Media (Springer, New York, 2006)

4. A. Shenoy, M.A. Sheremet, I. Pop, Convective flow and heat transfer from wavy surfaces: viscous fluids, porous media and nanofluids (CRC Press, Boca Raton, 2016)

5. A.A. Samarsky, Theory of difference schemes (Nauka, Moscow, 1977) 